\title{
Beam Walking to Assess Dynamic Balance in Health and Disease: A Protocol for the "BEAM" Multicenter Observational Study
}

\author{
Tibor Hortobágyi $^{a} \quad$ Azusa Uematsu $^{b} \quad$ Lianne Sanders $^{a} \quad$ Reinhold Kliegl ${ }^{c, d}$ \\ József Tolláre Renato Moraes ${ }^{f}$ Urs Granacher ${ }^{d}$ \\ ${ }^{a}$ Center for Human Movement Sciences, University of Groningen Medical Center, Groningen, The Netherlands; \\ ${ }^{b}$ Dokkyo Medical University, Shimotsuga-gun, Japan; ' Department of Psychology, University of Potsdam, Potsdam, \\ Germany; ${ }^{d}$ Division of Training and Movement Sciences, University of Potsdam, Potsdam, Germany; ${ }^{\text {e Department }}$ \\ of Neurology, Somogy County Kaposi Mór Teaching Hospital, Kaposvár, Hungary; ${ }^{f}$ School of Physical Education and \\ Sport of Ribeirão Preto, São Paulo, Brazil
}

\section{Keywords}

Aging $\cdot$ Gait $\cdot$ Balance $\cdot$ Dual tasks $\cdot$ Falls

\begin{abstract}
Background: Dynamic balance keeps the vertical projection of the center of mass within the base of support while walking. Dynamic balance tests are used to predict the risks of falls and eventual falls. The psychometric properties of most dynamic balance tests are unsatisfactory and do not comprise an actual loss of balance while walking. Objectives: Using beam walking distance as a measure of dynamic balance, the BEAM consortium will determine the psychometric properties, lifespan and patient reference values, the relationship with selected "dynamic balance tests," and the accuracy of beam walking distance to predict falls. Methods: This cross-sectional observational study will examine healthy adults in 7 decades $(n=432)$ at 4 centers. Center 5 will examine patients $(n=100)$ diagnosed with Parkinson's disease, multiple sclerosis, stroke, and balance disorders. In test 1, all participants will be measured for demographics, medical history, muscle strength, gait, static balance, dynamic balance using beam walking under single (beam walking only)
\end{abstract}

and dual task conditions (beam walking while concurrently performing an arithmetic task), and several cognitive functions. Patients and healthy participants age 50 years or older will be additionally measured for fear of falling, history of falls, miniBESTest, functional reach on a force platform, timed up and go, and reactive balance. All participants age 50 years or older will be recalled to report fear of falling and fall history 6 and 12 months after test 1 . In test 2 , seven to ten days after test 1 , healthy young adults and age 50 years or older $(n=40)$ will be retested for reliability of beam walking performance. Conclusion: We expect to find that beam walking performance vis-à-vis the traditionally used balance outcomes predicts more accurately fall risks and falls. Clinical Trial Registration Number: NCT03532984.

$$
\begin{aligned}
& \text { () } 2018 \text { The Author(s) } \\
& \text { Published by S. Karger AG, Basel }
\end{aligned}
$$

\section{Introduction}

Aging is associated with late-life mobility disability [1]. The walking speed becomes slower, steps become shorter and variable, and balance while standing and

\begin{tabular}{|c|c|}
\hline KARGER & $\begin{array}{l}\text { (c) } 2018 \text { The Author(s) } \\
\text { Published by S. Karger AG, Basel }\end{array}$ \\
\hline $\begin{array}{l}\text { E-Mail karger@karger.com } \\
\text { www.karger.com/ger }\end{array}$ & $\begin{array}{l}\text { This article is licensed under the Creative Commons Attribution- } \\
\text { NonCommercial-NoDerivatives } 4.0 \text { International License (CC BY- } \\
\text { NC-ND) (http://www.karger.com/Services/OpenAccessLicense). } \\
\text { Usage and distribution for commercial purposes as well as any dis- } \\
\text { tribution of modified material requires written permission. }\end{array}$ \\
\hline
\end{tabular}
walking becomes unstable, especially when these mobil-
Tibor Hortobágy

Center for Human Movement Sciences, University of Groningen Medical Center Antonius Deusinglaan 1

NL-9713 AV Groningen (The Netherlands)

E-Mail t.hortobagyi@umcg.nl 
ity tasks are combined with a motor or a cognitive task [2]. Gait, balance impairments and muscle weakness are associated with falls in $\sim 30$ and $\sim 50 \%$ of adults age over 65 and 80 years, respectively [3]. Nearly $50 \%$ of outdoor falls occur while walking [4]. Measuring dynamic balance is thus important to characterize the current state of postural control and identify those who are likely to experience severe levels of mobility disability, including falls in the future.

Dynamic balance is the maintenance of equilibrium while walking with and without a self- or external perturbation and resisting internal or external perturbations while standing [5]. In particular, dynamic balance keeps the vertical projection of the center of mass (COM) within the base of support while the COM moves during walking. To date, dynamic balance has been inferred from "functional tests" without an actual balance loss [6].

Functional tests measure dynamic balance indirectly and many suffer from a ceiling or floor effect and are insensitive to interventions and clinical status. The Berg Balance Scale (BBS) [7], designed for frail old adults, suffers from poor sensitivity to treatment effects and it has no common interpretation for a given score, unclearly related to mobility status and to the use of mobility aids. The Tinetti Balance and Gait Test [8] is a "functional test" used for "dynamic balance" but it identified only $11 \%$ of nonfallers, suggesting a poor specificity [6]. The TimedUp-and-Go (TUG) test [9] does not inform clinicians if patients' static or dynamic balance or walking ability is poor and it predicts falls inconsistently. Standing on one leg is difficult for many old adults and is unrelated to gait stability. Functional reach [10] has no walking element and patients compensate for balance problems during walking. The BESTest is organized around the systems underlying balance control and, similar to the Physiological Profile Approach, it takes over 30 min to administer; however, it has good reliability and a higher sensitivity to change than the BBS [11]. Its short version, i.e., the miniBESTest, takes only $\sim 15$ min to administer and has good reliability and discriminative validity between fallers and nonfallers [12], yet the reported scores vary widely and suggest a floor effect in patients with a spinal cord injury, a traumatic brain injury, an acquired brain injury, and multiple traumas [13]. Static posturography measures static balance and correlates poorly with dynamic balance [14]. The Star Excursion Balance Test, the Modified Bass Test, and the Dynamic Leap and Balance Test all involve unnatural movements or rapid changes of direction which seniors never perform. Dynamic posturography normally refers to responses to a perturbation given in standing or walking [15]. The face validity of the stability margin or the medial acceleration of the COM and the relationship between these variables and walking balance is unclear. Time-to-stabilization tests involve a single jump landing on the floor, which is unsuitable for seniors and patients. These latter tests and those measuring center of pressure (COP) outcomes require a force platform, making the tests unsuitable for clinicians. Inertial sensors can quantify walking balance during TUG but the analysis of gait dynamics requires sophisticated software and a highly specialized expertise to interpret the data [16].

\section{Beam Walking Performance to Measure Dynamic Balance in Old Age}

The single most important limitation of "dynamic balance" tests is a failure to quantify an actual balance loss while walking. Conventional functional tests of dynamic balance rely on gait speed to determine the fall risk. However, the balance element of walking is not related necessarily to the velocity of the COM but rather to the control of the small changes in acceleration of the COM caused by minute instabilities while walking [17]. The control of these accelerations makes balance highly specific and its assessment is insufficient through general mobility tests relying solely on gait speed [18]. Thus, a test that assesses a loss of balance during walking provides additional information on the intrinsic fall risk compared with tests that do not assess balance loss during walking and they rely on speed alone. In addition, gait speed can predict reasonably accurately falls in frail older adults but it is not as sensitive or accurate in healthier old adults [19]. Healthy old adults who walk $\geq 1.0 \mathrm{~m} / \mathrm{s}$ can have subclinical neuromuscular and cognitive problems masked by a gross measure such as gait speed, which a more challenging and balance-specific task could amplify. Indeed, pilot data revealed that while the habitual walking speed was similar in young and healthy old adults, the difference in beam walking performance was substantial (see pilot data).

Currently there is no laboratory-based or clinical test to assess a loss of dynamic balance during walking, impairment of balance during walking, or fall risk in a clinical setting. Beam walking could measure dynamic balance more accurately than tests currently in use because the reduction in base of support transiently increases instability associated with the movement of the COM over the stance leg. Beam compared with floor walking increases the challenge to dynamic balance [20] because the performer must control the path of the COM to not cross the edge of the foot support to stay on the beam. Such 
crossing often happens in old adults and a misstep, i.e., a "fall," ensues [21]. The instability during COM transfer while transiently on one leg and on a narrow beam is the key and unique element of beam walking that could amplify (sub)clinical dysfunctions in dynamic balance more effectively than current tests and make beam walking performance a novel biomarker of dynamic balance in health and disease.

\section{Application of Beam Walking in Patients with \\ Neurological Disorders}

Dysfunction in dynamic balance is a precursor to the high incidence of falls in neurological patients and identifying fall-related risk factors is a priority. Patients with Parkinson's disease (PD), multiple sclerosis (MS), and stroke had suffered one fall (47\%) or multiple falls (32\%) at the 6-month follow-up [22]. While the etiology leading to a fall differs between these patient categories, dysfunction of dynamic balance is a key common contributing element to mobility disability. Remarkably, disease type and balance confidence only and none of the "dynamic balance tests" or "functional tests" (BBS, Dynamic Gait Index, TUG, and 10-min walk test) predicted single and recurrent falls. There is thus a need to determine if beam walking compared with the currently used tests of dynamic balance is more accurate in diagnosing the fall risk and predicting falls in these patients.

\section{Cognitive Dual Tasking during Beam Walking}

Even though the gait speed slows with aging, implying an impaired dynamic balance, the conscious, cognitive, and cortical control of walking still increases [23]. In particular, when old adults couple walking with a cognitive task such as talking, calculation, or memory recall, the demand for attention and executive function increases to control the gait. When dual tasking, old adults' steps become variable and the gait stability decreases [2].

\section{Aims and Hypotheses}

This study will determine: (1) reliability and (2) lifespan and patient reference values for dynamic balance as determined by beam walking with and without cognitive dual-tasking, (3) the statistical relationship between dynamic balance (i.e., beam walking performance) and selected "functional tests" currently used to measure dynamic balance, and (4) the risk factors and predictors of falls in neurological patients. The global hypothesis is that age, disease type, and cognitive dual tasking affect dynamic balance as measured by beam walking performance (distance, velocity, and step number). In healthy
Table 1. Study design

\begin{tabular}{cll}
\hline & Centers 1-4 & Center 5 \\
\hline Subjects & Healthy & $\begin{array}{l}\text { PD, MS, stroke, } \\
\text { balance disorders }\end{array}$ \\
\hline Test & All subjects will be tested & $\begin{array}{l}\text { All subjects will } \\
\text { be tested }\end{array}$ \\
1 & A subsample will be retested & \\
2 & for dynamic balance & \\
\hline $\begin{array}{c}\text { Follow-up (months) } \\
6\end{array}$ & $\begin{array}{l}\text { Fall history at age } 50 \text { or older } \\
\text { Fall history at age } 50 \text { or older }\end{array}$ & $\begin{array}{l}\text { Fall history } \\
\text { Fall history }\end{array}$ \\
\hline
\end{tabular}

old adults, determinants of single and dual task beam walking performance are expected to include age, sex, executive function, physical activity, leg strength, and oneleg COP variability. In patients with neurological disorders, we expected that disease type, balance confidence, and executive function affect the single-task beam performance. Because of the novelty, it is unclear if patients can perform dual-task beam walking. We expect to find that beam walking performance vis-à-vis the traditionally used balance outcomes predicts more accurately fall risks and falls.

\section{Methods}

Design

This is a 5-site, multicenter cross-sectional observational study using the STROBE checklist (Table 1). Centers 1-4 will examine healthy old adults $(n=432)$ and center 5 will examine patients with a diagnosis of $\mathrm{PD}, \mathrm{MS}$, stroke, and nonspecific balance disorders $(n=100)$. Online supplement 1 (for all online suppl. material, see www.karger.com/doi/10/1159/000493360) shows the distribution of participants and tests. Selected healthy participants will repeat only the beam walking measurements at test 2 , seven to 10 days after test 1 , to assess reliability $(n=40)$. All participants age 50 years or older [24] will report their fall history at test 1 and at 6- and 12-months follow-ups. The primary outcome for all of the participants is beam-walking performance in meters. Age, sex, cognitive function, physical activity, and leg strength are determinants of dynamic balance and thus the sample size must be set to meet the recommendation of at least 4-5 times more participants than predictors. To increase external validity, universal reference values should be broad and representative of the heterogeneity of the population, which will be achieved by testing healthy male and female participants in 4 countries on 3 continents.

\section{Participants}

Healthy participants will be recruited from local areas at the 4 centers using word of mouth and advertisements in public areas, 
libraries, churches, newspapers, fitness clubs, and health care facilities, and on the radio, television, and internet. Male and female volunteers aged over 20 years will have good health, balance, and mobility. A positive answer to any of the following questions in a (phone) interview results in exclusion from this study: unable to walk $10 \mathrm{~m}$ independently; knee or hip joint replacements $\leq 6$ months before enrollment; uncontrolled cardiovascular disease or angina; neuromuscular disease; diagnosed PD, MS, or stroke; cancer therapy $\leq 3$ months before enrollment; severe asthma or chronic bronchitis; and diagnosed diabetes with neuropathy, poor and uncorrected vision, and a score $\leq 27$ on the Mini-Mental State Examination (MMSE). At the start of the laboratory visit the participants will perform the Short Physical Performance Battery (SPPB) and those healthy adults with a score $\leq 10$ for mobility will be excluded [25].

Center 5 (Kaposvár, Hungary) will recruit patients from the hospital's outpatient day clinic and medical database. Patients who report with balance and mobility difficulties and are candidates for or are currently enrolled in rehabilitation will be eligible. Patients with PD (Hoehn-Yahr stage 2-3) will meet the UK Brain Bank criteria. Patients with MS will meet the McDonald criteria of the International Panel on Diagnosis of MS. Patients with stroke will meet World Health Organization diagnostic criteria for stroke. Balance disorder patients who visit the outpatient clinic due to a fall and dizziness will be included. Excluded will be those with MMSE <21, major depression (Clinically Useful Depression Outcome score $\geq 46$ ) [26], severe joint and/or bone disorders interfering with balance and gait (clinical judgment), aphasia if it interferes with comprehension of the aims of this study, MS relapse within 3 months, stroke $<1$ month before the start of this study, benign paroxysmal positional vertigo, polyneuropathy, and phobic dizziness. Ethical committees at each site will approve the study protocol and the consent form, which each participant will read and sign. The study will be conducted according to the Declaration of Helsinki.

\section{Preliminary Data}

Healthy old $(n=16)$ and young $(n=20)$ volunteers walked on aluminum beams (length: $4 \mathrm{~m}$, height: $2 \mathrm{~cm}$, and widths: 4, 8, 12 $\mathrm{cm}$ ) with and without performing a calculation task [27]. The mean distance young participants walked decreased similarly with decreasing beam widths while single tasking $(12 \mathrm{~cm}: 3.88 \mathrm{~m}, 8 \mathrm{~cm}$ : $3.62 \mathrm{~m}$, and $4 \mathrm{~cm}: 2.49 \mathrm{~m})$ and dual tasking $(12 \mathrm{~cm}: 3.87 \mathrm{~m}, 8 \mathrm{~cm}$ : $3.76 \mathrm{~m}$, and $4 \mathrm{~cm}: 2.59 \mathrm{~m})$. The mean distance old adults walked decreased substantially and the most on the narrowest beam while single tasking ( $12 \mathrm{~cm}: 3.85 \mathrm{~m}, 8 \mathrm{~cm}: 3.72 \mathrm{~m}$, and $4 \mathrm{~cm}: 1.46 \mathrm{~m}$ ) but it decreased even more on the 2 narrowest beams during dual tasking ( $12 \mathrm{~cm}: 3.91 \mathrm{~m}, 8 \mathrm{~cm}: 2.63 \mathrm{~m}$, and $4 \mathrm{~cm}: 0.66 \mathrm{~m})($ age $\times$ beam width $\times$ task interaction, $F=4.0, p=0.026$ ). Beam width but not dual tasking affected young adults' dynamic balance, whereas both beam width and dual tasking affected substantially and interactively old adults' dynamic balance as quantified by beam walking performance. In contrast to the large differences in beam walking performance, $4-\mathrm{m}$ habitual walking speeds were not different $(p>$ 0.05 ) between the 2 age groups (young: $1.21 \pm 0.39$; old: $1.17 \pm 0.40$ $\mathrm{m} / \mathrm{s})$.

\section{Measurements}

Demographics will include age, height, foot length, foot width, body mass, sex, education, occupation, medications, and marital status. In all participants age 50 years or older, the presence of diagnosed diabetes, heart failure, hypertension, angina, myocardial infarction, chronic lung disease, urinary incontinence, depression, atrial fibrillation, a hearing aid, visual problems, arthritis, past cancers, present cancers, use of walking aids, and history and severity of falls (with and without complications) in the preceding 12 months will be recorded.

Physical activity will be estimated by the international physical activity questionnaire in all of the participants [28]. All tests will be conducted barefoot to rule out effects of footwear.

Fall history will be determined by having participants aged 50 years or older report falls for the previous 12 months ("Have you experienced a fall over the past 12 months? "How severe was the most severe fall? (hospitalization, fracture, nursing home admission)." Patients' hospital fall records will be also consulted if available. During the follow-up period, participants will record their falls daily on a scorecard ("Did you experience a fall today?" If yes, "How many times did you fall today? How severe was each fall?")

The Falls Efficacy Scale-International (FES-I) will determine in participants aged 50 years or older the level of concern about falling during social and physical activities indoors and outdoors. The internal validity of the FES-I is Cronbach's $\alpha=0.96$ and the testretest reliability is ICC $=0.96$ [29].

Balance confidence will be assessed with the Activities-specific Balance Confidence (ABC) Scale.

The Mini-BESTest identifies systems that control (dynamic) balance, differentiates between PD fallers and nonfallers (sensitivity: $62 \%$, specificity: $74 \%$ ), and is the most accurate among balance screens [30].

Mobility will be measured in all participants by the SPPB, a composite mobility test. The battery measures balance, 4-m habitual gait speed, and leg strength with good day-to-day reliability (ICC >0.80) [25].

Static steady-state balance will be assessed in all participants using modified the Romberg and sharpened Romberg tests while the patient is standing on a force platform with feet closed while holding both arms extended to the front with palms facing upwards and in tandem stance with eyes open and closed for $10 \mathrm{~s}$ for each task. The outcome is COP variability [31].

Static steady-state balance will be assessed by the modified Romberg test [32]. The outcomes are standing time (s) and the path velocity $(\mathrm{mm} / \mathrm{s})$ of the center of force.

Proactive (anticipatory) balance will be measured in all participants ages 20-29 and 50 or more years by FR while standing on a force platform, giving the outcomes of reach distance for each arm and COP variability and by TUG, providing a timed proactive balance outcome [31].

Reactive balance will be measured in all participants age 20-29 and 50 or more years by the push-and-release test with a score for the number of steps to regain balance after induction of balance loss [31].

Dynamic balance will be determined in all participants during beam walking [27]. The aluminum beams are $4 \mathrm{~m}$ long, $2 \mathrm{~cm}$ high, and 4,8 , and $12 \mathrm{~cm}$ wide, covered with slip resistance material, and placed on a thin, black rubber mat (online suppl. 2). After a practice trial, participants will perform 3 trials on each width with and without a calculation task (subtraction by 7 between 300 and 900 ). Trials will be block randomized based on beam width and then on task (calculation, no calculation). To reduce the risk for a 
fall, a technician will walk behind the participants on the floor. Instructions will be: "Traverse the entire length of the beam safely at your preferred speed without stepping off, facing forward, and with your arms folded in front of your chest. Trials end when you step off, walk sideways, or unfold the arms." Foot placement style and speed will not be controlled. Outcomes are the sum of length traversed ( 3 trials, $4 \mathrm{~m}$ each, $12 \mathrm{~m}$ maximum), average velocity, number of steps, and average step length determined from video tape recordings $(60 \mathrm{~Hz})$ made at $90^{\circ}$ relative to the walking direction with full beam length in view using off-the-shelf $\mathrm{HD}$ video cameras (Kinovea software). Two observers will also visually observe each trial from each side and measure the length, time (stop watch), and step number. They will mark immediately on the beam the heel of the foot that remained on the beam where balance was lost.

To determine dual-task costs for walking, the control condition is walking on a $4-\mathrm{m}$ long, $4-\mathrm{cm}$ wide, line taped on the floor at habitual speed 3 times with and without the calculation task. To determine dual-task costs for the cognitive task, the control condition is calculation in sitting for the average dual-tasking duration on the beams. Based on motor and cognitive dual-task costs, we determine if participants prioritize either task on the beam.

Upper extremity strength will be measured in all participants based on the grip strength of each hand.

Lower-extremity strength of the knee extensors will be measured in all participants in the seated position on a dynamometer or by a hand-held dynamometer affixed to the legs of the chair at ankle height.

Global cognition will be measured in all participants by the MMSE.

Attention and executive function will be measured in all participants by the Stroop color-word test [33] and phonemic fluency, which respectively measure selective attention, response interference, and executive processing. In the Stroop word condition (1), participants read the names of 4 colors (red, yellow, blue, and green). In the color condition (2), participants name these colors. In the interference condition (3), participants name the color of words printed in incongruent colors. The time to complete each condition (s) is recorded. An interference quotient is obtained by dividing the scores on condition 2 by those on condition 3 . In the phonemic fluency test, participants name as many words as possible that start with a certain letter. The total number of correct responses within $1 \mathrm{~min}$ is recorded.

Processing speed and intelligence fluidity will be measured in all participants by the Digit Symbol Substitution Test [34]. Participants match a series of symbols with their corresponding numbers 1 through 9 . The number of correct responses within $90 \mathrm{~s}$ is recorded.

Verbal and visual memory span and working memory. Memory spans and working memory will be measured in all participants by the Digit Span (DS) and Visual Memory Span (VMS) Forward and Backward [34]. In the DS, participants repeat a sequence of verbally presented digits in the same (DS forward) or reversed (DS backward) order. In the VMS, participants point to a sequence of squares in the presented (VMS forward) or reversed (VMS backward) order. The number of digits or squares increases after 2 trials. The total number of correct responses is recorded.

Follow-up measurements at 6 and 12 months will include a selfreported fear of falling and fall history.

\section{Statistical Analysis}

A manual of all tests and procedures will ensure consistent administration of the measurements at the centers.

Online supplement 3 shows the power analysis and power simulations in detail. Considering dropouts from the repeat measures and incomplete tests, we will include 48 (i.e., 24 males and $24 \mathrm{fe}$ males) healthy old participants per decade with a bias toward participants in their 60s $(n=80), 70 \mathrm{~s}(n=80)$, and $80 \mathrm{~s}(n=80)$ in anticipation of losing 30\% of participants at 6 and 12 months of follow-up (online suppl. 1 and 3). We understand that it may not be possible to achieve 80 participants aged 80 or older. We estimate that for the critical age-related 3-way interactions the power will average around $70 \%$ (online suppl. 3, bottom). We will include a convenience sample of 100 patients and expect to find a fall rate of $50 \%$ for the 6 months prior to and the 12 months after admission [22]. Thus, we expect an overall fall rate of approximately $40 \%$ for all participants age 65 years or older.

For aim 1, psychometric properties of beam walking will include: (1) the interrater reliability of the same 20 trials videotaped and evaluated by 2 different assessors for distance walked as the outcome (between centers); (2) the interrater reliability of the same 20 trials measured by 2 onsite assessors based on the visually measured beam walking distance at one location (within center); (3) the test-retest reliability of beam walking distance, step length, and step number based on the video-taped data; (4) the test-retest reliability of beam walking performance based on the visually measured distance, and (5) the accuracy of the visually measured beam walking distance relative to the distance measured using videotaped trials.

For aim 2, reference values of beam walking performance will be expressed as means, SD, coefficient of variation, ranges, and confidence intervals stratified by age and gender in healthy old adults. Such data will be derived for all and for subgroups of patients with PD, MS, stroke, and balance disorders. We will use an age $(7$ decades $) \times$ gender $(2) \times$ beam width $(3) \times$ task $($ single, dual $)$ analysis of variance to determine the effects of these factors on beam walking performance in terms of height-normalized distance, velocity, height-normalized stride length, and step number (normalized for distance walked). Foot width will be used as a covariate. Similar analyses will be done for all and the subgroups of patients. We will compare patients' performance with age- and gender-matched healthy adults using a health status (healthy, patient $) \times$ age $(60-69,70-79$, and 80 or more years $) \times$ beam width $\times$ task analysis of variance. Dual-task cost will be computed as ([single task - dual task]/single task) $\times 100$.

For aim 3, we will determine the relationship between dynamic balance, quantified by beam walking performance, and COP variability during static steady-state balance, proactive balance (FR in $\mathrm{cm}$ and TUG in s), and reactive balance (number of steps) in response to induction of a balance loss. Using stepwise forward moving multiple regression, we will identify the demographic, motor, and cognitive determinants of dynamic balance as measured by beam walking performance. In patients, we determine if disease type, balance confidence, and executive function affect single-task beam performance and compare outcome variables between those who report one fall, multiple falls, and no falls.

For aim 4, we will determine cumulative time-dependent probabilities of falls, recurrent falls, and injurious falls using KaplanMeier survival tables and curves in all healthy and patient participants combined and patients separately. Survival curves will be 
compared between groups using the log-rank test. The incidence ratio (i.e., the ratio between the total number of falls and the person-months at risk) will be also calculated. Multivariate Cox proportional hazards models will be used to identify independent predictors of falls, recurrent falls, and injurious falls. Univariate models will be used to select the predictors to be entered into multivariable models and only variables resulting significant in univariate models at $p<0.10$ will be entered in multivariable models. Risks will be computed as hazards ratios with $95 \%$ CI. $p<0.05$ was considered statistically significant.

\section{Discussion}

Dynamic balance is a determinant of mobility. A direct assessment of dynamic balance in gerontology is lacking, as it is indirectly inferred from walking speed [27] and qualitative self-reports [30]. The search and need for a valid and reliable measure of dynamic balance, i.e., an actual loss of balance, is highlighted by the use of an extraordinary array of 133 balance outcomes in 80 studies [35]. The difficulty in identifying a dynamic balance test may be related to the paradox that the number of falls increases despite reductions in walking speed. Indeed, the safety factor actually decreases with decreasing walking speed in old age [36]. Further, while walking slowly can increase the risk of falling backwards, the step length also becomes shorter and the COM moves closer to the base of support at toe off, which in turn reduces the risk of a backward fall [37]. Such compensations may mask underlying gait instabilities and make current tests to predict future falls insufficiently accurate. Thus, gait speed may not be an optimal index for dynamic balance.

If the results of the present protocol bear out, beam walking, which produces a discrete endpoint for dynamic balance as a result of an actual loss of balance, could be conceptually well founded yet a practical test of dynamic balance [38-40]. While the validity of the beam walking test cannot be established due to the lack of a gold standard, correlations between beam-walking performance and the miniBESTest, the SPPB, and the TUG can help determine if beam walking measures unique features of dynamic balance. Beam walking is a challenging task because it requires active and conscious control of mediolateral stability [41]. An increase in trunk stabilization in space contributes substantially to reducing the difficulty of beam walking as shown by use of the anchors [20]. Beam walking differentiated between old adults with and without a cognitive problem, attesting to the presence of a cognitive element in beam walking [42]. It was especially sensitive to exercise effects in healthy old adults [43].
There is much debate as to how gait slowing is related to gait stability and falls. While there is evidence suggesting that age-associated gait slowing is an independent predictor of falls, others interpret gait slowing as an adaptive mechanism to prevent slips and falls, which would in turn reduce the risk of falls [44]. Still others suggest that not gait speed per se but rather the quality of the gait is what increases the prediction accuracy for future falls [45], and using dynamic systems analyses some but not all studies found that a slow gait is actually more stable [46]. The present study would help clarify these issues by increasing the specificity of balance prediction from a performance that is quantified by an actual loss of balance while walking on a low-lying beam and measure walking speed on a beam and also on the floor.

We are unaware of any studies measuring dynamic balance through beam walking in patients with neurological and balance disorders. With a high rate of falls among these patients, we will determine and compare the sensitivity, specificity, and predictive accuracy of the miniBESTest, the SPPB, and the TUG and, as a new test, beam walking performance. If successful, beam walking would offer clinicians a new reliable and sensitive test to assess dynamic balance in a broad range of patients.

In sum, we will determine the psychometric properties, lifespan, and patient reference values for dynamic balance quantified by beam walking performance, its relationship with "functional tests" currently used to measure dynamic balance, and its accuracy in predicting falls in fall-prone patients. We expect to find that beam walking performance vis-à-vis the traditionally used balance outcomes predicts more accurately fall risks and falls.

\section{Disclosure Statement}

The authors declare that they have no competing interests.

\section{Funding Sources}

This work was supported in part by: JSPS KAKENHI grant No. 16K21320 (Japan), a startup fund from the University Medical Center Groningen (653013; The Netherlands) and the German Research Foundation (Deutsche Forschungsgemeinschaft, DFG, priority program SPP 1772: grants GR 3997/4-1, HE 7464/1-1, and RA 1047/4-1). The funders had no role in the study design, data collection and analysis, the decision to publish, or the preparation of this paper. 


\section{References}

1 Studenski S, Perera S, Patel K, Rosano C, Faulkner K, Inzitari M, Brach J, Chandler J, Cawthon P, Connor EB, Nevitt M, Visser M, Kritchevsky S, Badinelli S, Harris T, Newman AB, Cauley J, Ferrucci L, Guralnik J: Gait speed and survival in older adults. JAMA 2011;305:50-58.

2 Beauchet O, Dubost V, Herrmann FR, Kressig RW: Stride-to-stride variability while backward counting among healthy young adults. J Neuroeng Rehabil 2005;2:26.

3 Rubenstein LZ: Falls in older people: epidemiology, risk factors and strategies for prevention. Age Ageing 2006;35(suppl 2):ii37ii41.

4 Li W, Keegan TH, Sternfeld B, Sidney S, Quesenberry CP Jr, Kelsey JL: Outdoor falls among middle-aged and older adults: a neglected public health problem. Am J Public Health 2006;96:1192-1200.

5 Ringhof S, Stein T: Biomechanical assessment of dynamic balance: specificity of different balance tests. Hum Mov Sci 2018;58:140-147.

6 Mancini M, Horak FB: The relevance of clinical balance assessment tools to differentiate balance deficits. Eur J Phys Rehabil Med 2010; 46:239-248.

7 Berg KO, Maki BE, Williams JI, Holliday PJ, Wood-Dauphinee SL: Clinical and laboratory measures of postural balance in an elderly population. Arch Phys Med Rehabil 1992;73 1073-1080.

8 Tinetti ME, Speechley M: Prevention of falls among the elderly. N Engl J Med 1989;320: 1055-1059.

9 Podsiadlo D, Richardson S: The timed "Up and Go": a test of basic functional mobility for frail elderly persons. J Am Geriatr Soc 1991; 39:142-148.

10 Duncan PW, Weiner DK, Chandler J, Studenski S: Functional reach - a new clinical measure of balance. J Gerontol 1990;45:M192M197.

11 Godi M, Franchignoni F, Caligari M, Giordano A, Turcato AM, Nardone A: Comparison of reliability, validity, and responsiveness of the mini-BESTest and Berg Balance Scale in patients with balance disorders. Phys Ther 2013;93:158-167.

12 Potter K, Brandfass K: The Mini-Balance Evaluation Systems Test (Mini-BESTest). J Physiother 2015;61:225.

13 Roaldsen KS, Wakefield E, Opheim AP: Pragmatic evaluation of aspects concerning validity and feasibility of the Mini Balance Evaluation System Test in a specialized rehabilitation hospital. Int J Phsy Ther Rehab 2015;1: 104.

14 Granacher U, Bridenbaugh SA, Muehlbauer T, Wehrle A, Kressig RW: Age-related effects on postural control under multi-task conditions. Gerontology 2011;57:247-255.
15 Hof AL, Vermerris SM, Gjaltema WA: Balance responses to lateral perturbations in human treadmill walking. J Exp Biol 2010;213: 2655-2664.

16 Kikkert LHJ, de Groot MH, van Campen JP, Beijnen JH, Hortobagyi T, Vuillerme N, Lamoth CCJ: Gait dynamics to optimize fall risk assessment in geriatric patients admitted to an outpatient diagnostic clinic. PLoS One 2017;12:e0178615.

17 Fujimoto M, Chou LS: Sagittal plane momentum control during walking in elderly fallers. Gait Posture 2016;45:121-126.

18 Shumway-Cook A, Woollacott MH: Motor Control, ed 5. Philadelphia, Wolters Kluwer, 2017.

19 Montero-Odasso M, Schapira M, Soriano ER, Varela M, Kaplan R, Camera LA, Mayorga LM: Gait velocity as a single predictor of adverse events in healthy seniors aged 75 years and older. J Gerontol A Biol Sci Med Sci 2005; 60:1304-1309.

20 Costa A, Santos LOD, Mauerberg-deCastro E, Moraes R: Task difficulty has no effect on haptic anchoring during tandem walking in young and older adults. Neurosci Lett 2018; 666:133-138.

21 Chiu MC, Wu HC, Chang LY, Wu MH: Center of pressure progression characteristics under the plantar region for elderly adults. Gait Posture 2013;37:408-412.

22 Beghi E, Gervasoni E, Pupillo E, Bianchi E, Montesano A, Aprile I, Agostini M, Rovaris M, Cattaneo D: Prediction of falls in subjects suffering from Parkinson disease, multiple sclerosis, and stroke. Arch Phys Med Rehabil 2018;99:641-651.

23 Kikkert LHJ, Vuillerme N, van Campen JP, Hortobagyi T, Lamoth CJ: Walking ability to predict future cognitive decline in old adults: a scoping review. Ageing Res Rev 2016;27:114.

24 Hall KS, Cohen HJ, Pieper CF, Fillenbaum GG, Kraus WE, Huffman KM, Cornish MA, Shiloh A, Flynn C, Sloane R, Newby LK, Morey MC: Physical performance across the adult life span: correlates with age and physical activity. J Gerontol A Biol Sci Med Sci 2017;72:572-578.

25 Guralnik JM, Simonsick EM, Ferrucci L, Glynn RJ, Berkman LF, Blazer DG, Scherr PA, Wallace RB: A short physical performance battery assessing lower extremity function: association with self-reported disability and prediction of mortality and nursing home admission. J Gerontol 1994;49:M85-M94.

26 Zimmerman M, Chelminski I, McGlinchey JB, Posternak MA: A clinically useful depression outcome scale. Compr Psychiatry 2008; 49:131-140

27 Uematsu A, Tsuchiya K, Yokei H, Suzuki S, Hortobágyi T: Cognitive dual-tasking augments age-differences in dynamic balance during beam walking. Exp Gerontol, in press.
28 Craig CL, Marshall AL, Sjostrom M, Bauman AE, Booth ML, Ainsworth BE, Pratt M, Ekelund U, Yngve A, Sallis JF, Oja P: International physical activity questionnaire: 12-country reliability and validity. Med Sci Sports Exerc 2003;35:1381-1395.

29 Yardley L, Beyer N, Hauer K, Kempen G, Piot-Ziegler C, Todd C: Development and initial validation of the Falls Efficacy Scale-International (FES-I). Age Ageing 2005;34:614619.

30 Yingyongyudha A, Saengsirisuwan V, Panichaporn W, Boonsinsukh R: The Mini-Balance Evaluation Systems Test (Mini-BESTest) demonstrates higher accuracy in identifying older adult participants with history of falls than do the BESTest, Berg Balance Scale, or Timed Up and Go Test. J Geriatr Phys Ther 2016;39:64-70.

31 Gschwind YJ, Kressig RW, Lacroix A, Muehlbauer T, Pfenninger B, Granacher U: A best practice fall prevention exercise program to improve balance, strength/power, and psychosocial health in older adults: study protocol for a randomized controlled trial. BMC Geriatr 2013;13:105.

32 Lacroix A, Kressig RW, Muehlbauer T, Gschwind YJ, Pfenninger B, Bruegger O, Granacher $\mathrm{U}$ : Effects of a supervised versus an unsupervised combined balance and strength training program on balance and muscle power in healthy older adults: a randomized controlled trial. Gerontology 2016;62:275288

33 Stroop JR: Studies of interference in serial verbal reactions. J Exp Psychol 1935;18:643-662.

34 Wechsler D: Wechsler Adult Intelligence Scale, ed 3. San Antonio, The Psychological Corporation, 1997.

35 Farlie MK, Robins L, Haas R, Keating JL, Molloy E, Haines TP: Programme frequency, type, time and duration do not explain the effects of balance exercise in older adults: a systematic review with a meta-regression analysis. Br J Sports Med 2018, DOI: 10.1136/bjsports-2016-096874.

36 Mademli L, Arampatzis A: Lower safety factor for old adults during walking at preferred velocity. Age (Dordr) 2014;36:9636.

37 Espy DD, Yang F, Bhatt T, Pai YC: Independent influence of gait speed and step length on stability and fall risk. Gait Posture 2010;32: 378-382.

38 Sawers A, Ting LH: Beam walking can detect differences in walking balance proficiency across a range of sensorimotor abilities. Gait Posture 2015;41:619-623.

39 Chiovetto E, Huber ME, Sternad D, Giese MA: Low-dimensional organization of angular momentum during walking on a narrow beam. Sci Rep 2018;8:95. 
40 Sipp AR, Gwin JT, Makeig S, Ferris DP: Loss of balance during balance beam walking elicits a multifocal theta band electrocortical response. J Neurophysiol 2013;110:2050-2060.

41 Arvin M, Mazaheri M, Hoozemans MJM, Pijnappels M, Burger BJ, Verschueren SMP, van Dieen JH: Effects of narrow base gait on mediolateral balance control in young and older adults. J Biomech 2016;49:1264-1267.

42 Alexander NB, Mollo JM, Giordani B, Ashton-Miller JA, Schultz AB, Grunawalt JA, Foster NL: Maintenance of balance, gait pat- terns, and obstacle clearance in Alzheimer's disease. Neurology 1995;45:908-914.

43 Buchner DM, Cress ME, de Lateur BJ, Esselman PC, Margherita AJ, Price R, Wagner EH: A comparison of the effects of three types of endurance training on balance and other fall risk factors in older adults. Aging (Milano) 1997;9:112-119.

44 Quach L, Galica AM, Jones RN, Procter-Gray E, Manor B, Hannan MT, Lipsitz LA: The nonlinear relationship between gait speed and falls: the Maintenance of Balance, Indepen- dent Living, Intellect, and Zest in the Elderly of Boston Study. J Am Geriatr Soc 2011;59: 1069-1073.

45 van Schooten KS, Pijnappels M, Rispens SM, Elders PJ, Lips P, Daffertshofer A, Beek PJ, van Dieen JH: Daily-life gait quality as predictor of falls in older people: a 1-year prospective cohort study. PLoS One 2016; 11:e0158623.

46 Bruijn SM, van Dieen JH, Meijer OG, Beek PJ: Is slow walking more stable? J Biomech 2009; 42:1506-1512. 